\title{
Trimethylamine-N-Oxide Aggravates Kidney Injury via Activation of p38/MAPK Signaling and Upregulation of HuR
}

\author{
Yunshi Lai Haie Tang Xinrong Zhang Zhanmei Zhou Miaomiao Zhou \\ Zheng Hu Fengxin Zhu Lei Zhang Jing Nie
}

State Key Laboratory of Organ Failure Research, National Clinical Research Center of Kidney Disease, Key Laboratory of Organ Failure Research (Ministry of Education), Guangdong Provincial Clinical Research Center for Kidney Disease, Division of Nephrology, Nanfang Hospital, Southern Medical University, Guangzhou, PR China

\section{Keywords}

Trimethylamine-N-oxide · Inflammatory injury · p38/MAPK pathway · Human antigen $\mathrm{R} \cdot$ Chronic kidney disease

\begin{abstract}
Background: Trimethylamine-N-oxide (TMAO) is an intestinal metabolic toxin, which is produced by gut flora via metabolizing high-choline foods. TMAO is known to increase the risk of atherosclerosis and cardiovascular events in chronic kidney disease (CKD) patients. Objectives: The objective of this study was to explore the role and mechanism of TMAO aggravating kidney injury. Method: We used the five-sixths nephrectomy (5/6 Nx)-induced CKD rats to investigate whether TMAO could aggravate kidney damage and its possible mechanisms. Six weeks after the operation, the two groups of $5 / 6 \mathrm{Nx}$ rats were subjected to intraperitoneal injection with $2.5 \%$ glucose peritoneal dialysis fluid $(2.5 \%$ PDF) and 2.5\% PDF plus TMAO $20 \mathrm{mg} / \mathrm{kg} /$ day. Results: In this study, we provided evidence showing TMAO significantly aggravated renal failure as well as inflammatory cell infiltration and in five-sixths nephrectomy-induced CKD rats. We found that TMAO could upregulate inflammatory factors including MCP-1, TNF-a, IL-6, IL-1 $\beta$, and IL-18 by activating p38
\end{abstract}

karger@karger.com www.karger.com/kbr

Karger"
(C) 2021 The Author(s)

Published by S. Karger AG, Basel

This is an Open Access article licensed under the Creative Commons Attribution-NonCommercial-4.0 International License (CC BY-NC) (http://www.karger.com/Services/OpenAccessLicense), applicable to the online version of the article only. Usage and distribution for commercial purposes requires written permission. phosphorylation and upregulation of human antigen $\mathrm{R}$. TMAO could aggravate oxidative stress by upregulating NOX4 and downregulating SOD. The result also confirmed that TMAO promoted NLRP3 inflammasome formation as well as cleaved caspase- 1 and IL-1 $\beta$ activation in the kidney tissue. Conclusions: Taken together, the present study validates TMAO as a pro-inflammatory factor that causes renal inflammatory injury and renal function impairment. Inhibition of TMAO synthesis or promoting its clearance may be a potential therapeutic approach of CKD in the future.

(c) 2021 The Author(s).

Published by S. Karger AG, Basel

\section{Introduction}

Chronic kidney disease (CKD) is a worldwide public health problem leading to end-stage renal disease (ESRD) [1]. Chronic inflammation is considered as a major driving force of CKD progression. The causes of systemic inflammation in CKD patients include direct induction by uremic toxins, oxidative stress, and microbial infections.

Yunshi Lai and Haie Tang contributed equally to this work.
Correspondence to:

Lei Zhang, zhanglei_nfyy@163.com

Jing Nie, niejing@smu.edu.cn 
Inflammation may interfere with intrarenal microcirculation regulation and recruit inflammatory cells to further aggravate renal injury. Several cytokines such as IL-1, IL6 , and TNF- $\alpha$ have been demonstrated to increase significantly in CKD patients. Numerous clinical studies have demonstrated that high TNF- $\alpha$ and IL- 6 levels are closely associated with poor prognostic of CKD, such as large amount of albuminuria and progressive loss of renal function $[2,3]$.

The gut microbiota imposes a crucial effect on regulating mucosal immunity. CKD causes an imbalance of the intestinal flora and leads to immune disorders [4]. Previous studies have shown that the intestinal flora of CKD patients is significantly decreased in probiotics, while increased in toxigenic flora [5]. Imbalance of the gut flora and dysfunction of the intestinal barrier lead to translocation of intestinal flora. Their toxic products also transfer into the host circulatory system and lead to persistent systemic inflammation. Intestinal toxins indoxyl sulfate and p-Cresyl sulfate have been demonstrated to promote inflammatory macrophage activation [6] and increase production of pro-inflammatory cytokines [7].

Uremic toxins may also promote the imbalance between formation of reactive oxygen species (ROS) and antioxidant capacity, which leads to excessive oxidative stress. Increased ROS can induce inflammation, endothelial dysfunction, atherosclerosis and fibrosis, and is considered to be a powerful promoter of CKD [8]. Active NADPH oxidases (NOX) are dominant sources of ROS, and NOX4 is the major type of NOX in the kidney. Previous studies have demonstrated that intrarenal NOX4 contributes to immune-cell activation in the progression of CKD [9].

TMAO is produced by gut flora via metabolizing food containing choline, lecithin, betaine, and carnitine [10]. TMAO is predominantly excreted by the kidney, so its serum level is significantly increased in ESRD patients. It is in the spotlight recently because of its tight association with risk of cardiovascular events in both CKD and nonCKD patients $[11,12]$. Many studies have demonstrated that dietary choline or TMAO can promote inflammatory activation of vascular endothelial cells and platelets aggregation, leading to atherosclerosis and CVD events. It has been demonstrated that choline TMA-lyase inhibitor, IMC, can suppress renal tubulointerstitial fibrosis caused by high-choline diet [13]. However, whether TMAO contributes to renal interstitial inflammation remains unknown. In the present study, we explored the role of TMAO in inflammatory injury and the underlying mechanism in the CKD rat model.

\section{Method}

\section{Animal Experiments}

Male Sprague-Dawley rats (at the age of 5-6 weeks, initial weight 180-200 g; Southern Medical University Animal Experiment Center) were subdivided into 3 groups randomly, and 5 in each group. The first group received sham operation, while the other 2 groups received five-sixths nephrectomy (5/6 Nx) as described previously [14]. Six weeks after the operation, the two groups of 5/6 Nx rats were subjected to intraperitoneal injection with 2.5\% glucose peritoneal dialysis fluid (PDF; Baxter Health Care, Deerfield, IL, USA) and 2.5\% glucose PDF plus TMAO 20 $\mathrm{mg} / \mathrm{kg} /$ day (Sigma-Aldrich, St. Louis, MO, USA), respectively. The sham group was injected with PBS every day. Six weeks after injection, all the rats were sacrificed, and blood samples and kidney tissues were collected. Serum TMAO was detected by stable isotope dilution liquid chromatography tandem mass spectrometry (6460 Series Triple Quadrupole LC/MS; Agilent, Santa Clara, CA, USA).

\section{Histological and Immunohistochemistry Staining}

Paraffin-embedded kidney sections $(2 \mu \mathrm{m})$ were subjected to hematoxylin and eosin ( $\mathrm{H} \& \mathrm{E})$ and Masson trichrome staining according to standard protocols. Tubular injury was graded with $\mathrm{H} \& \mathrm{E}$-stained sections ranging from 0 to 4 according to the degree of tubular necrosis, dilatation, or cell swelling: 0 , less than $5 \%$; 1 , $5-25 \%$; 2, 25-50\%; 3, 50-75\%; and 4, over 75\% [15]. At least 10 randomly chosen fields in the cortex region under the microscope $(\times 400)$ were evaluated for each animal in a blinded manner, and an average score was calculated.

Interstitial fibrosis was assessed using Image-Pro Plus 6.0 (Media Cybernetics, Silver Spring, MD, USA) on Masson trichrome stained sections. Ten visual fields $(\times 400)$ were randomly selected for each animal and evaluated by a background subtraction method. Quantification is presented as the ratio of optical density of positive staining compared to the entire spectrum.

Immunohistochemistry staining was performed on $4-\mu \mathrm{m}$ kidney sections. After antigen retrieval, sections were incubated with the primary antibodies against CD68 (Abcam, Cambridge, UK) or CD3 (Abcam). Images were taken by an Olympus BX51 micro-

Fig. 1. TMAO injection promotes renal functional decline in 5/6-nephrectomized rats and aggravates renal tubular injury. Six weeks after five-sixths nephrectomy, the CKD rats were randomly divided into 2 groups and received daily intraperitoneal injection of either PDF containing 2.5\% glucose (2.5\% PDF) or $2.5 \%$ PDF plus TMAO (20 mg/kg/day), respectively. The sham group was injected with PBS every day. Blood samples and kidney tissues were collected $24 \mathrm{~h}$ after 5 weeks of injection. A-C Serum levels of TMAO, Scr, and BUN. D Representative images of H\&E and Masson's trichrome staining of the kidney tissue. Original magnification, $\times 400$; scale bar, $50 \mu \mathrm{m}$. Tubular injury score $(\mathbf{E})$ and quantification assessment of kidney fibrosis $(\mathbf{F})$. Bars depict mean values $\pm \mathrm{SD}$ ( $n=6$ per group). Results were analyzed for statistical variance using one-way ANOVA analysis. ${ }^{*} p<0.05$ versus sham group; ${ }^{\#} p<0.05$ versus $C K D$ injected $2.5 \%$ PDF group. PDF, peritoneal dialysis fluid; TMAO, trimethylamine-N-oxide; Scr, serum creatinine; BUN, blood urea nitrogen; CKD, chronic kidney disease; $H \& E$, hematoxylin and eosin.

(For figure see next page.)
62

Kidney Blood Press Res 2022;47:61-71 DOI: $10.1159 / 000519603$
Lai/Tang/Zhang/Zhou/Zhou/Hu/Zhu/ Zhang/Nie 
A

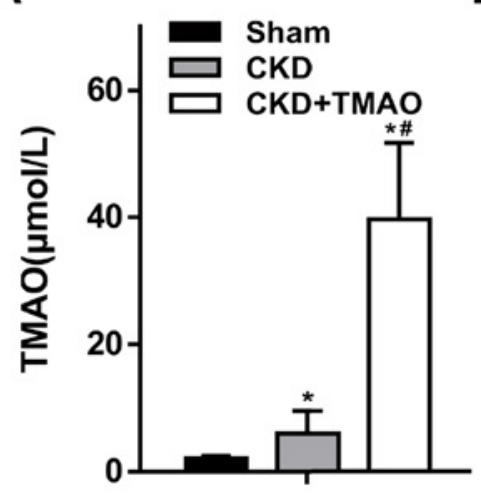

B

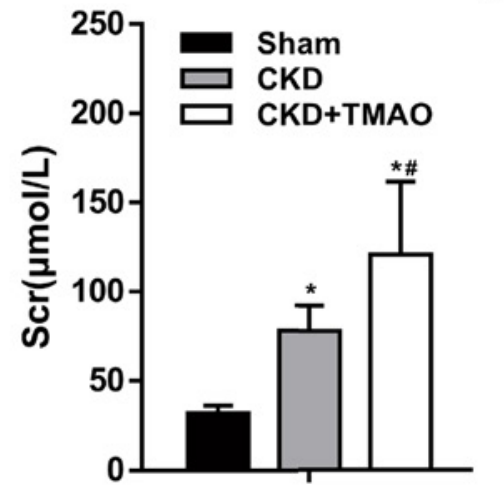

C

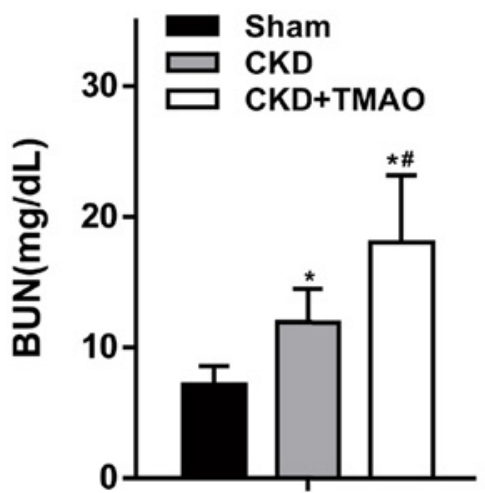

D

$\operatorname{Sham}(400 x)$

CKD(400x)

CKD+TMAO(400x)
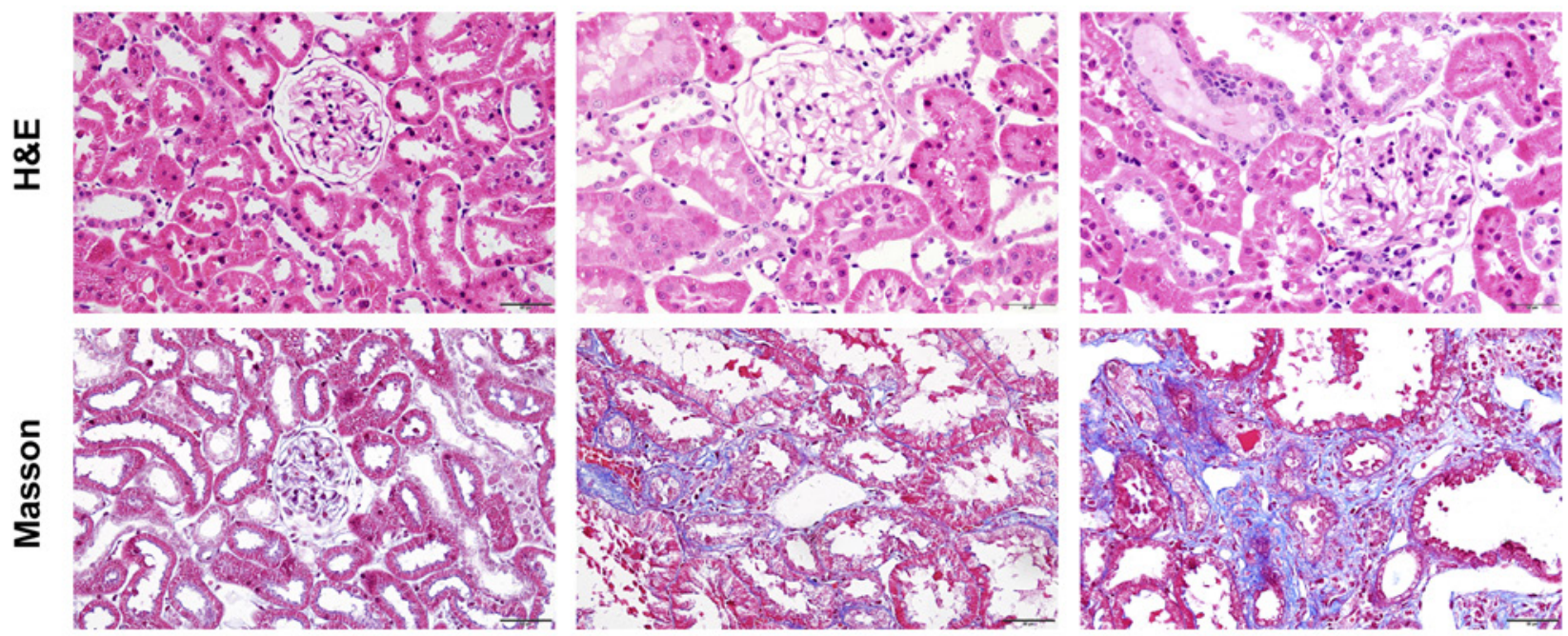

E

$\mathbf{F}$

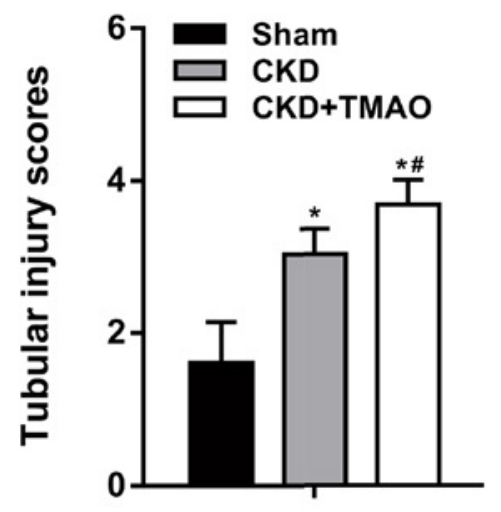


scope (Olympus, Tokyo, Japan). Positive staining cells were counted under microscope in 10 random fields $(\times 400)$ for each section.

\section{Western Blot Analysis}

Kidney tissues were lysed in lysis buffer for $30 \mathrm{~min}$ on ice. Western blot analysis was performed following procedure as described previously. The following primary antibodies were used: anti-IL-6 (Boster Biological Technology, Pleasanton, CA, USA), anti-IL-1 $\beta$ (Abcam), anti- $\beta$-actin (Boster Biological Technology), anti-human antigen R (HuR) (Santa Cruz, Santa Cruz, CA, USA), antiTTP (Abcam), anti-NLRP3 (Cell Signaling Technology, Beverly, MA, USA), anti-phosphorylated-p38 (Cell Signaling Technology), anti-p38 (Cell Signaling Technology), and anti-caspase-1 ( Santa Cruz).

\section{mRNA Analysis}

RNA isolation and real-time quantitative polymerase chain reaction were conducted as recommended by MIQE guidelines. Total RNA was extracted from homogenized kidney cortex tissue. TNF- $\alpha$, IL-6, IL-1 $\beta$, IL-18, and MCP-1 mRNA levels were quantified by real-time quantitative polymerase chain reaction with the Applied Biosystems 7500 Fast Real-Time PCR System (Foster City, CA, USA). Gene expression was normalized relative to the housekeeping gene, $\beta$-actin. Primers used in this study were listed in online supplementary Table 1 (for all online suppl. material, see www.karger.com/doi/10.1159/000519603).

Renal Primary Tubular Epithelial Cells Isolation and Culture Isolation of kidney primary tubular epithelial cells (PTECs) was conducted according to the methods described in the previous study [16]. Eight-week-old male C57BL/6 mice (Southern Medical University Animal Experiment Center) were used for PTEC isolation. Briefly, tissues were obtained from the kidney cortex and digested with collagenase IV solution until glomerulus and renal tubes were isolated. Subsequently, the digestion was stopped with equal volume of $10 \%$ FBS (Gibco) of PBS. Then, the solution was centrifuged and resuspended with $32 \%$ Percoll solution. Finally, the PTECs were cultured in DMEM/F12 (Gibco) medium supplemented with $10 \% \mathrm{FBS}, 100 \mathrm{U} / \mathrm{mL}$ penicillin, and $100 \mathrm{~g} / \mathrm{mL}$ streptomycin (Hyclone) at $37^{\circ} \mathrm{C}$ in a $5 \% \mathrm{CO}_{2}$ incubator.

\section{Dihydroethidium Fluorescence Analysis}

Primary renal tubular epithelial cells from C57BL/6 mice were cultured on 12 -well plates until they attained $80 \%$ confluency and then incubated with $25 \mathrm{~mm}$ glucose medium in the presence or absence of indicated concentration of TMAO for $24 \mathrm{~h}$. After $24 \mathrm{~h}$, all the cells were incubated with $3 \mu \mathrm{M}$ dihydroethidium (Beyotime Biotechnology, Shanghai, China) for $30 \mathrm{~min}$ at $37^{\circ} \mathrm{C}$ in a $5 \% \mathrm{CO}_{2}$ incubator. Fluorescence intensity was measured using flow cytometry (BD FACS; Calibur, San Francisco, CA, USA) at excitation/ emission filters of 488/575 $\mathrm{nm}$ [17].

\section{Statistical Analysis}

For in vivo study and in vitro experiments, data were expressed as means \pm SD. Results were analyzed for statistical variance using an unpaired Student's $t$ test or one-way ANOVA analysis where appropriate. A two-sided $p$ value $<0.05$ was considered to be statistically significant (SPSS software, version 20.0; SPSS, Inc., Chicago, IL, USA).

\section{Result}

\section{TMAO Aggravates Renal Tubule Injury and}

\section{Inflammatory Cells Infiltration}

Previous studies have shown that TMAO is excreted through the kidneys and that TMAO levels gradually increase with renal function decline. As expected, serum levels of TMAO were significantly increased in CKD rats compared with those of the sham group $(4.22 \pm 1.98 \mu \mathrm{M}$ vs. $2.14 \pm 0.34 \mu \mathrm{M}$ ), while those of TMAO group were prominently higher than those of the CKD group (39.72 $\pm 11.97 \mu \mathrm{M}$ vs. $4.22 \pm 1.98 \mu \mathrm{M}$ ) (Fig. 1A). The data also showed that serum creatinine and blood urea nitrogen were both significantly elevated in the TMAO group compared with the CKD group (Fig. 1B, C), indicating that TMAO could further accelerate CKD progression.

Then, we performed histopathological examination to assess renal pathological injury. $\mathrm{H} \& \mathrm{E}$ staining revealed that in the TMAO group, the vacuolar and necrotic degeneration of renal tubular epithelial cells as well as inflammatory cells infiltration were much more severe than those in the CKD group. Masson trichrome staining also showed that TMAO treatment significantly exacerbated renal interstitial fibrosis in CKD rats (Fig. 1D, F). When evaluated by tubular injury score, the TMAO group had a significantly higher score than those of the CKD and sham groups (Fig. 1E).

Further analysis by immunohistochemistry staining showed the infiltration of macrophages $\left(\mathrm{CD} 8^{+}\right.$cells $)$and lymphocytes $\left(\mathrm{CD}^{+}\right.$cells) in the tubulointerstitial area. The amounts of both types of cells were significantly increased in the CKD group compared with the sham group, while the TMAO treatment drove the trend even further (Fig. 2). Our observations revealed that TMAO could promote inflammatory cells infiltration and renal tubular injury, which lead to the decline of kidney function.

\section{TMAO Promotes Expression of Inflammatory Cytokines}

As chemotaxis of inflammatory cells plays a crucial role in inflammation, we detected MCP-1, a key chemotactic cytokine involved in the migration of circulating monocytes. The result showed that MCP-1 was significantly increased in renal tissue by TMAO treatment (Fig. 3A, B). Next, we compared levels of inflammatory cytokines including TNF- $\alpha$, IL-6, IL- $1 \beta$, and IL-18 in kidney tissue among the groups. It was found that these inflammatory cytokines except IL-18 were significantly raised in the kidney tissues of the TMAO treatment group compared with those of the CKD group (Fig. 3C). Col- 
A
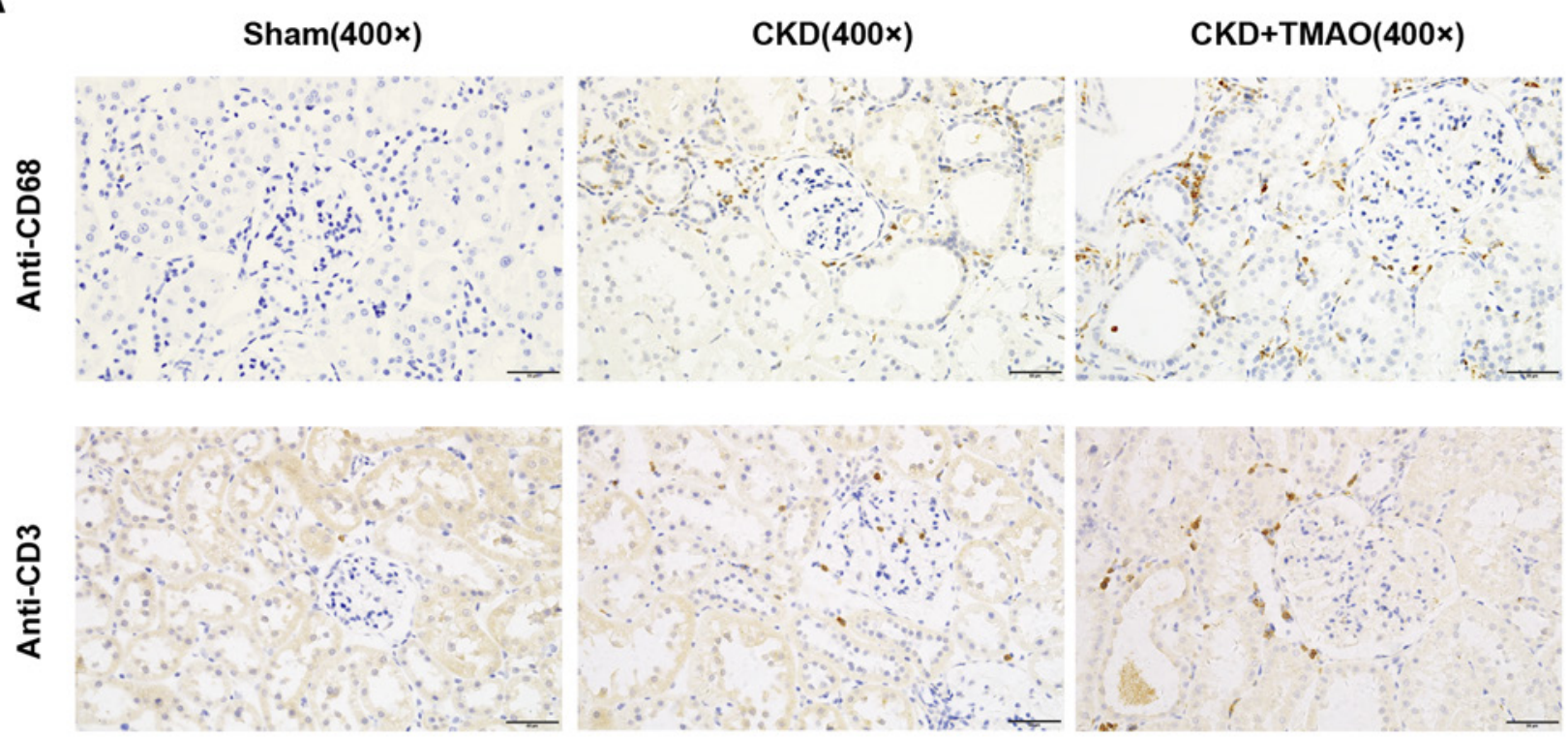

B
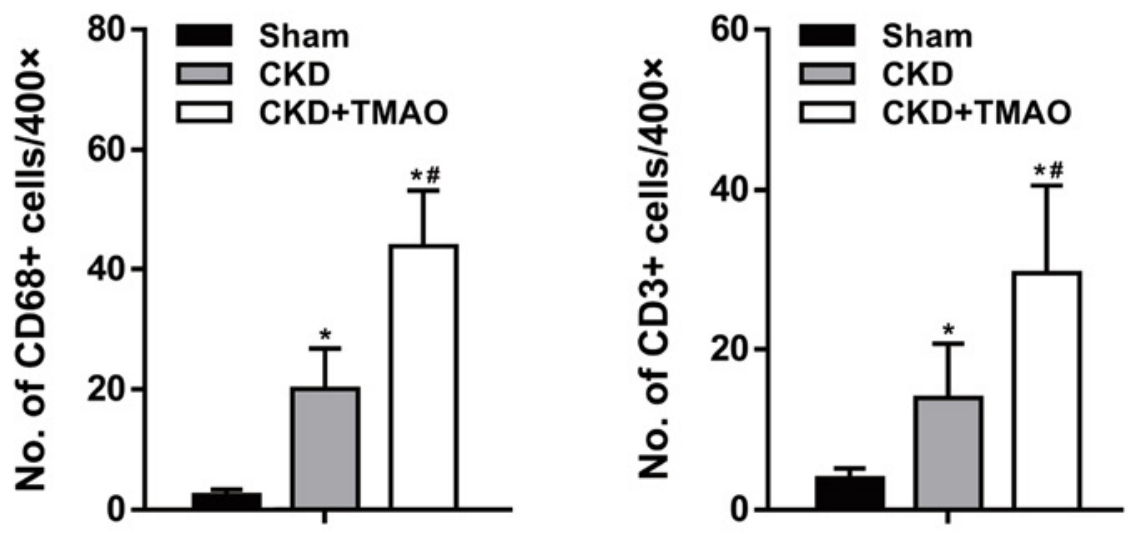

Fig. 2. TMAO aggravates $5 / 6 \mathrm{Nx}$-induced renal inflammation. A Representative images of immunohistochemical staining of $\mathrm{CD} 68^{+}$and $\mathrm{CD}^{+}$in the renal cortex. Bar, $50 \mu \mathrm{m}$. B Quantification assessment of positive $\mathrm{CD}^{+} 8^{+}$or $\mathrm{CD}^{+}$cells, respectively. Data were expressed as the mean $\pm \mathrm{SD}(n=5$ per group). Results were analyzed for statistical variance using one-way ANOVA analysis. ${ }^{*} p<0.05$ versus sham group; ${ }^{*} p<0.05$ versus CKD injected 2.5\% PDF. PDF, peritoneal dialysis fluid; TMAO, trimethylamine-N-oxide; CKD, chronic kidney disease.

lectively, these data demonstrated that TMAO could promote inflammatory cell chemotaxis and increase the expression of inflammatory cytokines.

TMAO Exacerbates the Activation of the p38/MAPK

Pathway Induced by 5/6 Nx

Then, we tried to analyze the signaling pathway through which TMAO promotes kidney inflammation.
First, we detected the NF- $\kappa \mathrm{B} / \mathrm{p} 65$ pathway, but the result did not support that TMAO increased the phosphorylation level of p65 in renal tissue (seen in online suppl. Fig. 1). Since the p38/MAPK pathway also plays a central role in the fibrosis process after inflammatory injury, we examined the level of phosphorylation of p38 as well as its downstream molecules. As is shown in (Fig. 4A, B), the ratio of phosphorylated $\mathrm{p} 38 / \mathrm{p} 38$ was elevated in $\mathrm{CKD}$ rats 
compared with that of the sham group, and TMAO injection promoted the trend even further. Phosphorylated p38 is known to activate MK2/3 phosphorylation, which

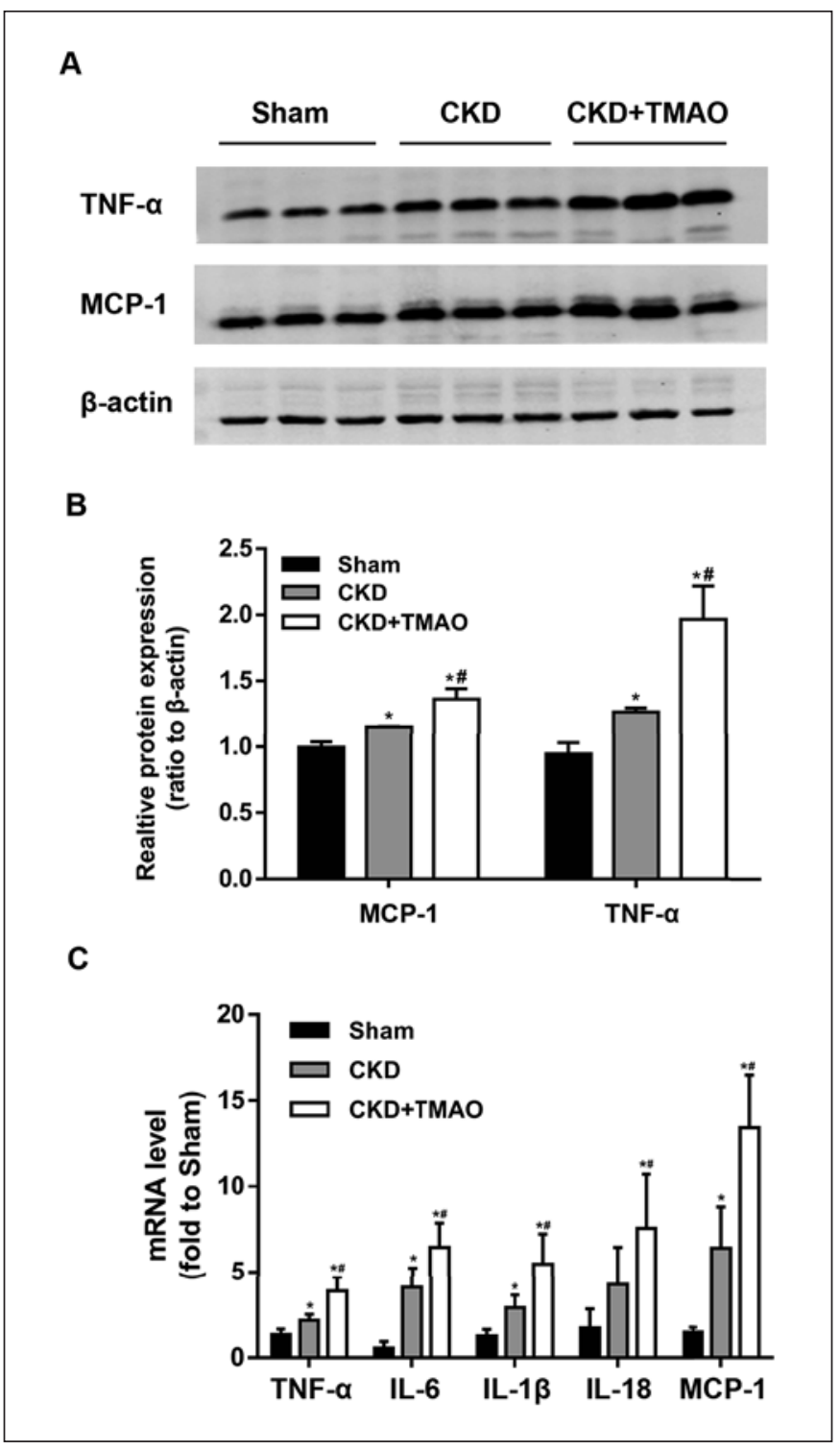

Fig. 3. TMAO promotes $5 / 6 \mathrm{Nx}$-induced inflammatory cytokines expression. A, B Protein levels of MCP-1 and TNF- $\alpha$ in the renal cortex were determined by Western blotting. $\beta$-Actin served as a loading control. Representative Western blot images and quantification of MCP-1 and TNF-a protein levels were shown. $\mathbf{C}$ mRNA levels of inflammatory markers including TNF- $\alpha$, IL-6, IL-1 $\beta$, IL18 , and MCP-1 were determined by real-time PCR. Data are expressed as the mean $\pm \mathrm{SD}$ ( $n=5$ per group). Results were analyzed for statistical variance using one-way ANOVA analysis. ${ }^{*} p<0.05$ versus sham group; ${ }^{\#} p<0.05$ versus $\mathrm{CKD}$ injected $2.5 \%$ PDF. TMAO, trimethylamine-N-oxide; CKD, chronic kidney disease; PDF, peritoneal dialysis fluid. can upregulate $\mathrm{HuR}$, while downregulate tristetraprolin (TTP) [18].

\section{TMAO Upregulates HuR while Downregulating TTP}

$\mathrm{HuR}$ is a type of RNA-binding protein that can combine with the AU-rich element (ARE) in the $3^{\prime}$ untranslated region ( $3^{\prime}$ UTR) of mRNA. It has been reported to maintain the mRNA stability of inflammatory cytokines such as TNF- $\alpha$, IL-6, and IL-18 [19, 20]. TTP is another type of RNA-binding protein. But, TTP can promote the degradation of these cytokines, and HuR can competitively inhibit TTP. Consistently, we observed that TMAO treatment could significantly upregulate $\mathrm{HuR}$ and downregulate TTP ( $p<0.05$ ) (Fig. 4C, D). Our findings may partially explain why TMAO can promote the mRNA and protein levels of the inflammatory cytokines.

\section{TMAO Increases NOX4 Expression and Aggravates \\ Oxidative Stress}

Upregulation of NOX4 is crucial in renal oxidative stress and kidney injury. As NOX4 mRNA also has numerous AREs in its $3^{\prime}$-UTR that can be bounded by HuR, we detected the expression of NOX4 in kidney tissue. The level of superoxide dismutase 2 (SOD2) in kidney tissue was also examined, because it is the major antioxidant enzyme catalyzing the dismutation of superoxide anions into oxygen and hydrogen peroxide [21]. The results showed that compared with the CKD group, TMAO treatment significantly raised the levels of NOX4 in kidney tissue, while pulled down the levels of SOD2 (Fig. 4E,

Fig. 4. TMAO activates the $5 / 6 \mathrm{Nx}$-induced P38/MAPK pathway and aggravates oxidative stress. A, B Protein levels of p-p38/p38 in the renal cortex were determined by Western blotting. The relative protein levels were expressed as the fold increase compared to that of the sham group in 3 independent experiments. C, D Representative Western blot images and quantification of protein levels of TTP and HuR in the renal cortex were presented. E, F Representative Western blot images and quantification of protein levels of NOX4 and SOD2 in the renal cortex. $\beta$-actin served as a loading control. Data are expressed as the mean $\pm \operatorname{SD}\left(n=5\right.$ per group). ${ }^{*} p$ $<0.05$ versus sham group; ${ }^{\#} p<0.05$ versus CKD injected $2.5 \%$ PDF. G Primary renal tubular epithelial cells of rats were cultured with high-glucose medium (glucose $25 \mathrm{mmol} / \mathrm{L}$ ) and then treated with gradient concentrations of TMAO $(0 \sim 400 \mu \mathrm{M})$. The levels of superoxides in these cells were detected by DHE fluorescence. Data are expressed as the mean \pm SD ( $n=5$ per group). Results were analyzed for statistical variance using one-way ANOVA analysis. ${ }^{*} p<0.05$ versus control; ${ }^{\#} p<0.05$ versus cells cultured with highglucose medium solely. HuR, human antigen R; TTP, tristetraprolin; DHE, dihydroethidium; TMAO, trimethylamine-N-oxide; $\mathrm{CKD}$, chronic kidney disease; PDF, peritoneal dialysis fluid.

(For figure see next page.)
66

Kidney Blood Press Res 2022;47:61-71 DOI: $10.1159 / 000519603$
Lai/Tang/Zhang/Zhou/Zhou/Hu/Zhu/ Zhang/Nie 
A

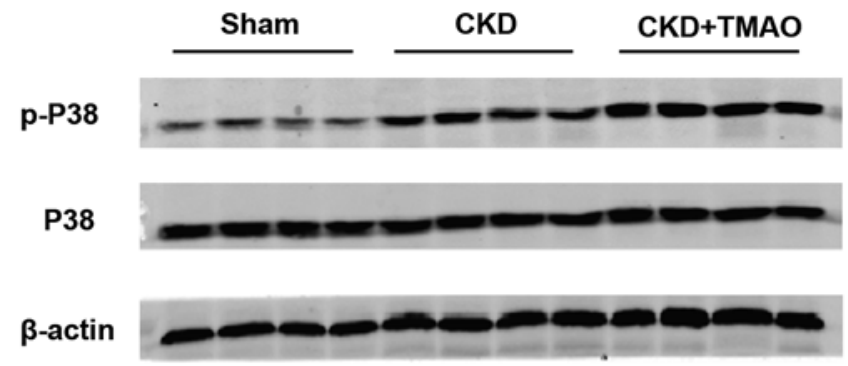

C

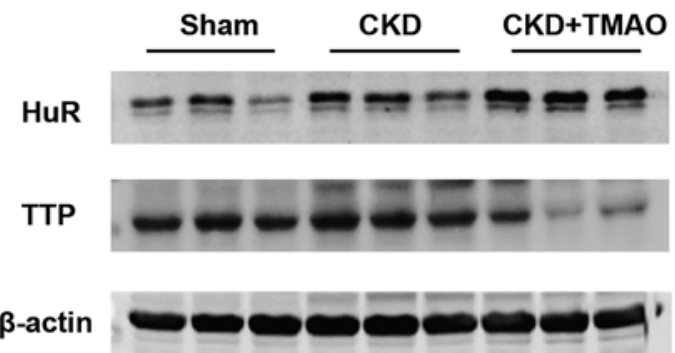

E

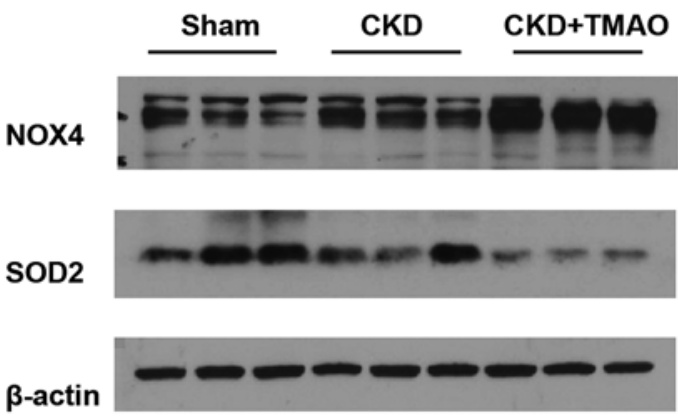

G
B

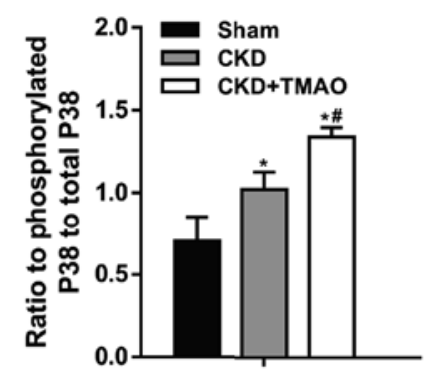

D

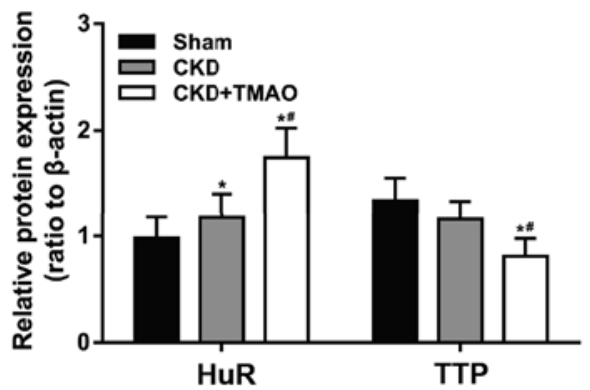

$\mathbf{F}$

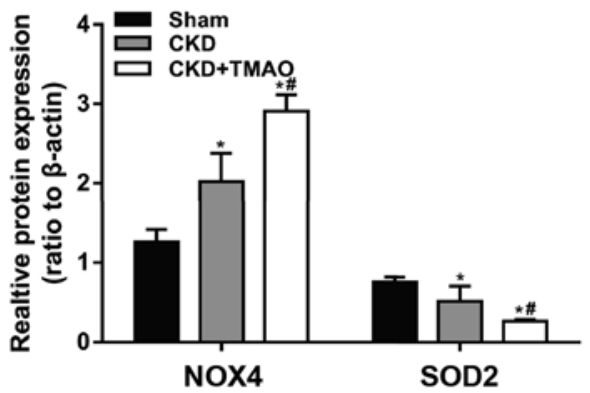

4

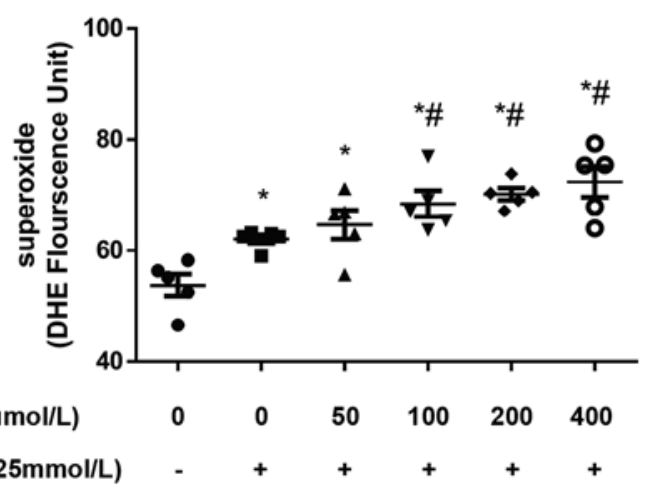


Fig. 5. TMAO activates $5 / 6 \mathrm{Nx}$-induced NLRP3 inflammasomes. A Representative Western blots showed the abundance of NLRP3, cleaved caspase-1, and cleaved IL$1 \beta$ in the renal cortex in sham rats and CKD rats injected with $2.5 \% \mathrm{PDF}$ or $2.5 \% \mathrm{PDF}$ plus TMAO. B Relative protein levels of NLRP3, cleaved caspase-1, and cleaved IL$1 \beta$ compared to $\beta$-actin. Data are expressed as the mean $\pm \mathrm{SD}$ ( $n=5$ per group). Data were analyzed for statistical variance using one-way ANOVA analysis. ${ }^{*} p<0.05$ versus sham group; ${ }^{*} p<0.05$ versus $C K D$ injected 2.5\% PDF. TMAO, trimethylamine-N-oxide; CKD, chronic kidney disease; PDF, peritoneal dialysis fluid.

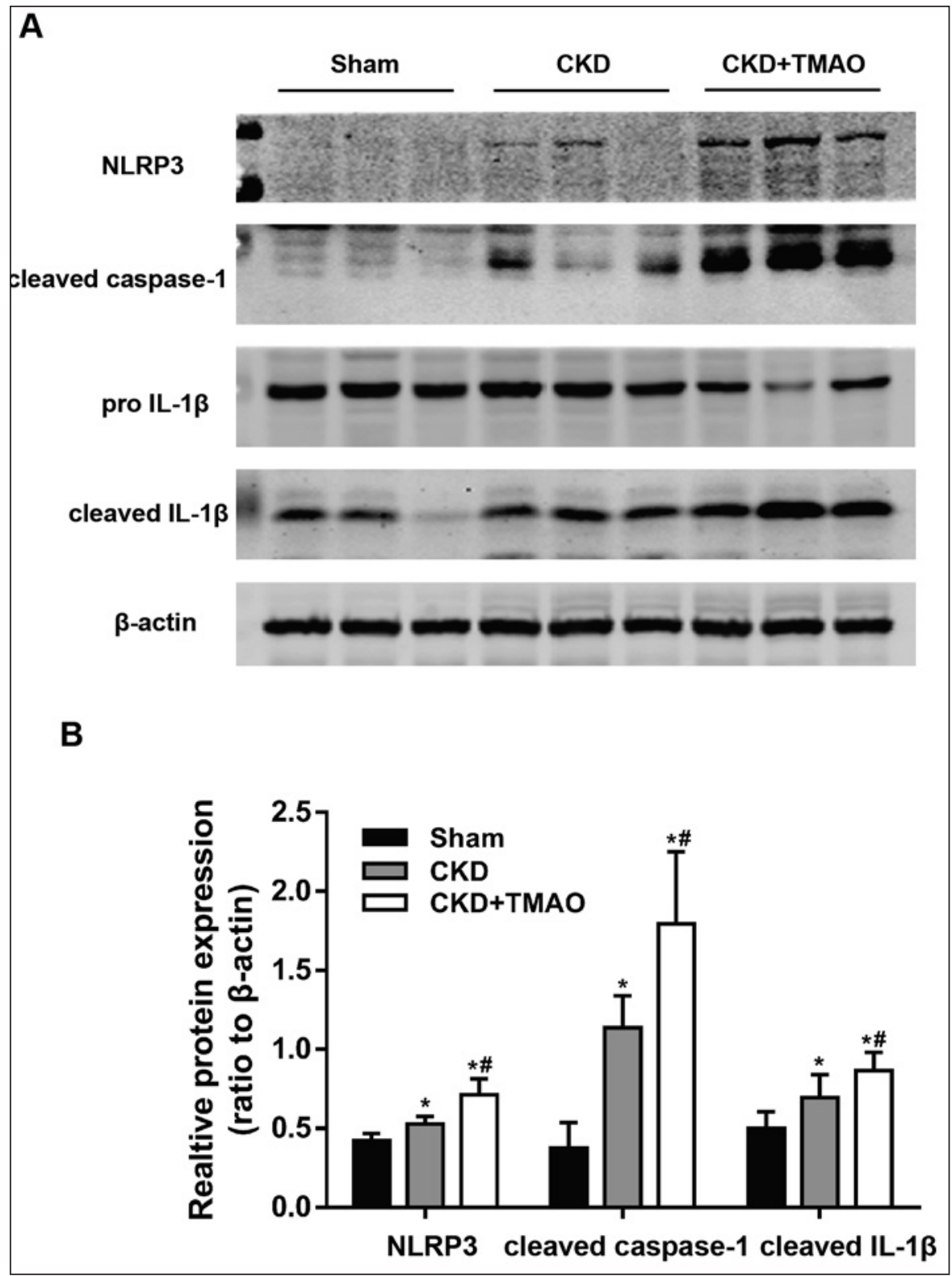

F). In addition, we tested the effect of TMAO on ROS production in renal tubular epithelial cells. High glucosecultured PTECs were stimulated with gradient concentrations of TMAO $(0 \sim 400 \mu \mathrm{M})$ for $24 \mathrm{~h}$. Dihydroethidium fluorescence analysis showed that levels of superoxide in renal tubular cells were significantly elevated by TMAO treatment (Fig. 4G). These data demonstrated that TMAO could aggravate oxidative stress in renal tissue.

\section{TMAO Promotes NLRP3 Inflammasomes Activation}

Inflammasomes turn oxidative stress signal signals into the release of inflammatory cytokines which rapidly set off local and systemic inflammation. We also assessed the levels of major members involved in the NOD-like receptor protein 3 (NLRP3) inflammasome pathway by Western blot. The result revealed that TMAO significantly elevated the protein levels of NLRP3, cleaved caspase-1, and cleaved IL- $1 \beta$ compared with the nontreated CKD group (Fig. 5). The data above demonstrated that TMAO could aggravate tubular inflammation via NLRP3 inflammasome activation.
68

Kidney Blood Press Res 2022;47:61-71 DOI: $10.1159 / 000519603$
Lai/Tang/Zhang/Zhou/Zhou/Hu/Zhu/ Zhang/Nie 


\section{Discussion}

TMAO, a metabolite of intestinal flora, has been recognized to promote atherosclerosis, but its role in CKD progression is still unknown. Based on previous studies, we explored the effects of TMAO on renal interstitial inflammation. There are several important findings in the present study. First, the study found that TMAO could significantly aggravate renal function decline and tubular interstitial injury. Second, TMAO could activate the inflammatory pathway through increasing p38 phosphorylation and HuR level. Last, TMAO upregulated NOX4 as well as promoted oxidative stress and NLRP3 activation.

Chemotaxis and infiltration of inflammatory cells is the key step of renal inflammatory injury [22]. We found that the infiltration of $\mathrm{CD}^{+} 8^{+}$and $\mathrm{CD}^{+}$cells were significantly increased in the TMAO group. TMAO treatment also stimulated the expression of MCP-1 as well as inflammatory factors including IL-6, IL- $1 \beta$, IL-18, and TNF- $\alpha$ in the kidney tissue. Consistently, the renal tubular injury score in the TMAO group was significantly higher than that in the CKD group. It is still controversial whether mature IL- $1 \beta$ can be secreted from renal intrinsic cells. Some studies suggest that IL- $1 \beta$ comes from activated macrophages. From the results above, we speculate that TMAO can induce renal tubular epithelial cell injury and recruit macrophages, which in turn release more inflammatory cytokines.

Then, we sought to identify the pathway by which TMAO activated the inflammatory signaling in the kidney. As is well known, p38 is the core component of the MAPK pathway, and phosphorylated p38 can turn on the transcription of many target genes, such as TNF- $\alpha$, MCP1 , and NLRP3 inflammasome [23, 24]. Our results revealed that TMAO had a promoting effect on p38 phosphorylation in the MAPK pathway but not on phosphorylation of either ERK or p65. Seldin et al. [25] has reported that TMAO could induce the MAPK signaling cascade in vascular endothelial cells in the model of aortic disease. p38/MAPK is also a major pro-inflammatory pathway for other intestinal flora toxins. For example, the uremia toxin p-cresyl sulfate is reported to activate JNK/ p38 MAPKs and stimulate ROS production [26].

In addition to promoting p38 phosphorylation, we found that TMAO could upregulate HuR and downregulate TTP in the kidney. Previous studies have shown that HuR could regulate several pro-inflammatory factors such as IL-6, TNF- $\alpha$, and C-reactive protein via binding to AREs in their $3^{\prime}$-UTR. One study in glioblastoma cells showed that IL- $1 \beta$ could enhance IL- 6 mRNA through

TMAO Aggravates Kidney Injury via p38/ MAPK and HuR the p38 MAPK-HuR pathway [27]. HuR increases in various kidney disease models and is involved in glomerulosclerosis. Another research revealed that inhibition of HuR could significantly ameliorate podocyte injury, macrophage cell infiltration as well as fibrogenic protein deposition [28]. As cytoplasmic localization of $\mathrm{HuR}$ is controlled by the p38/MAPK pathway, the results above support the speculation that TMAO indirectly promotes inflammation by regulating transcription factor $\mathrm{HuR}$ and TTP.

Our data also revealed that TMAO increased the NOX4 level, while decreased the SOD2 level in the kidney. It is known that NOX4 expression is increased in diabetic kidney disease, accompanied with ROS generation and mesangial-cell fibrotic injury [29]. NOX4 has numerous AREs in its $3^{\prime}$-UTR that can be bounded by HuR [30]. Shi et al. [30] observed that HuR could bind to the NOX4 promoter and increase the ROS level of mesangial cells in response to high glucose. Therefore, we hypothesize that TMAO could increase the NOX4 level by upregulating $\mathrm{HuR}$, thus aggravating oxidative stress in CKD. Since direct inhibition of oxidative stress and NOX4 inhibitors have not been clinically beneficial, inhibition of TMAO synthesis may be a feasible treatment to reduce oxidative stress and ameliorate CKD.

Another novelty of this study is that TMAO could promote NLRP3 inflammasome formation as well as caspase- 1 activation and IL- $1 \beta$ synthesis in the CKD model. It has been reported that TMAO promotes vascular calcification through activation of NLRP3 inflammasome and NF- $\kappa B$ signaling [31]. The mechanism by which TMAO activates NLRP3 remains unclear. In general, bacterial endotoxins stimulate inflammation via the toll-like receptor 4 and NLRP3 inflammasome pathways [32]. Oxidative stress is considered to be another inducer of NLRP3 inflammasome activation. It has also been demonstrated that NOX4 can downregulate the fatty-acid oxidation pathway by inhibiting carnitine palmitoyl transferase $1 \mathrm{~A}$ and then stimulates NLRP3 inflammasome activation and IL- $1 \beta$ production $[32,33]$. In summary, TMAO may activate the NLRP3-IL- $1 \beta$ axis, then promote inflammatory cell chemotaxis and inflammatory cytokine release, and finally lead to tubulointerstitial injury.

There are several limitations in the current study. Firstly, in order to directly test the effects of TMAO, rather than other intestinal metabolites from high-choline diet, intraperitoneal injection of TMAO was used in this study. Nevertheless, repeated injections may also increase the risk of peritoneal inflammation and intestinal damage. 
Secondly, we did not collect the urine for urinary albumin measurement, which is also an important indicator for CKD model. Finally, in addition to the effect of TMAO on the protein levels of HuR and TTP, the impact of TMAO on their function and the combination with downstream genes should also be explored in future studies.

\section{Conclusion}

We provide evidence showing that in the CKD rat model, TMAO could regulate the expression of downstream inflammatory factors by activating $\mathrm{p} 38$ phosphorylation and upregulation of HuR. Activation of inflammatory pathways results in the upregulation of NOX4 and activation of NLRP3 inflammasome in kidney tissue. The increasing inflammation cytokines and oxidative stress aggravate inflammatory cell infiltration, and eventually lead to renal function deterioration. TMAO may become a therapeutic target to ameliorate renal tubular interstitial injury and delay the progression of CKD.

\section{Acknowledegment}

Dr. Boxin Zhao is acknowledged for performing the serum TMAO quantification.

\section{Statement of Ethics}

This study protocol was reviewed and approved by the Nanfang Hospital Animal Ethic Committee, approval number NFYY-2017118.

\section{Conflict of Interest Statement}

We declare that we have no financial and personal relationships with other people or organizations that can inappropriately influence our work; there is no professional or other personal interest of any nature or kind in any product, service, and/or company that could be construed as influencing the position presented in, or the review of, the manuscript entitled "Trimethylamine-N-oxide aggravates kidney injury via activation of p38/MAPK signaling and upregulation of HuR."

\section{Funding Sources}

This work was supported by grants from the Medical Science and Technology Research Foundation of Guangdong Province (A2020292) and the President Foundation of Nanfang Hospital, Southern Medical University (2017C051), to Dr. Lei Zhang and by the National Natural Science Foundation of China (81730019, $81521003)$ to Dr. Jing Nie.

\section{Author Contributions}

Lei Zhang and Jing Nie conceived and designed the research; Yunshi Lai and Haie Tang performed the animal experiments and analyzed the data; Xinrong Zhang, Fengxin Zhu, and Zheng $\mathrm{Hu}$ helped to conduct CKD animal models. Zhanmei Zhou and Miaomiao Zhou directed the preparation of histopathological sections. Jing Nie and Lei Zhang wrote the paper.

\section{Data Availability Statement}

The data that support the findings of this study are openly available in figshare at 10.6084/m9.figshare.14703138.

\section{References}

1 Zhang L, Wang F, Wang L, Wang W, Liu B, Liu J, et al. Prevalence of chronic kidney disease in China: a cross-sectional survey. Lancet. 2012;379:815-22.

2 Upadhyay A, Larson MG, Guo CY, Vasan RS, Lipinska I, O'Donnell CJ, et al. Inflammation, kidney function and albuminuria in the Framingham Offspring cohort. Nephrol Dial Transplant. 2011;26:920-6.

3 Amdur RL, Feldman HI, Gupta J, Yang W, Kanetsky P, Shlipak M, et al. Inflammation and progression of CKD: the CRIC study. Clin J Am Soc Nephrol. 2016;11:1546-56.

4 Andersen K, Kesper MS, Marschner JA, Kon$\operatorname{rad} \mathrm{L}$, Ryu M, Kumar Vr S, et al. Intestinal dysbiosis, barrier dysfunction, and bacterial translocation account for CKD-related systemic inflammation. J Am Soc Nephrol. 2017; 28:76-83.
5 Sabatino A, Regolisti G, Brusasco I, Cabassi A, Morabito S, Fiaccadori E. Alterations of intestinal barrier and microbiota in chronic kidney disease. Nephrol Dial Transplant. 2015;30: 924-33.

6 Nakano T, Katsuki S, Chen M, Decano JL, Halu A, Lee LH, et al. Uremic toxin indoxyl sulfate promotes proinflammatory macrophage activation via the interplay of OATP2B1 and Dll4-Notch signaling. Circulation. 2019;139:78-96.

7 Matsuo K, Yamamoto S, Wakamatsu T, Takahashi Y, Kawamura K, Kaneko Y, et al. Increased proinflammatory cytokine production and decreased cholesterol efflux due to downregulation of ABCG1 in macrophages exposed to indoxyl sulfate. Toxins. 2015;7: 3155-66.
8 Duni A, Liakopoulos V, Roumeliotis S, Peschos D, Dounousi E. Oxidative stress in the pathogenesis and evolution of chronic kidney disease: untangling ariadne's thread. Int $\mathrm{J} \mathrm{Mol}$ Sci. 2019;20:3711.

9 Kumar V, Kurth T, Zheleznova NN, Yang C, Cowley AW. NOX4/H2O2/mTORC1 pathway in salt-induced hypertension and kidney injury. Hypertension. 2020;76:133-43.

10 Koeth RA, Wang Z, Levison BS, Buffa JA, Org E, Sheehy BT, et al. Intestinal microbiota metabolism of L-carnitine, a nutrient in red meat, promotes atherosclerosis. Nat Med. 2013;19:576-85.

11 Shafi T, Powe NR, Meyer TW, Hwang S, Hai $\mathrm{X}$, Melamed ML, et al. Trimethylamine N-oxide and cardiovascular events in hemodialysis patients. J Am Soc Nephrol. 2017;28:321-31. 
12 Tang WH, Wang Z, Kennedy DJ, Wu Y, Buffa JA, Agatisa-Boyle B, et al. Gut microbiotadependent trimethylamine $\mathrm{N}$-oxide (TMAO) pathway contributes to both development of renal insufficiency and mortality risk in chronic kidney disease. Circ Res. 2015;116: 448-55.

13 Gupta N, Buffa JA, Roberts AB, Sangwan N, Skye SM, Li L, et al. Targeted inhibition of gut microbial trimethylamine $\mathrm{N}$-Oxide production reduces renal tubulointerstitial fibrosis and functional impairment in a murine model of chronic kidney disease. Arterioscler Thromb Vasc Biol. 2020;40:1239-55.

14 Gschwend S, Buikema H, Navis G, Henning RH, de Zeeuw D, van Dokkum RP. Endothelial dilatory function predicts individual susceptibility to renal damage in the $5 / 6$ nephrectomized rat. J Am Soc Nephrol. 2002;13: 2909-15.

15 Leemans JC, Stokman G, Claessen N, Rouschop KM, Teske GJ, Kirschning CJ, et al. Renal-associated TLR2 mediates ischemia/reperfusion injury in the kidney. J Clin Invest. 2005;115:2894-903.

16 Qu Y, An F, Luo Y, Lu Y, Liu T, Zhao W, et al. A nephron model for study of drug-induced acute kidney injury and assessment of druginduced nephrotoxicity. Biomaterials. 2018; 155:41-53.

17 Qian Y, Zhang Y, Zhong P, Peng K, Xu Z, Chen $X$, et al. Inhibition of inflammation and oxidative stress by an imidazopyridine derivative $\mathrm{X} 22$ prevents heart injury from obesity. J Cell Mol Med. 2016;20:1427-42.

18 Ronkina N, Shushakova N, Tiedje C, Yakovleva T, Tollenaere MAX, Scott A, et al. The role of TTP phosphorylation in the regulation of inflammatory cytokine production by MK2/3. J Immunol. 2019;203:2291-300.
19 Liu Y, Wei W, Wang Y, Wan C, Bai Y, Sun X, et al. TNF- $\alpha$ /calreticulin dual signaling induced NLRP3 inflammasome activation associated with HuR nucleocytoplasmic shuttling in rheumatoid arthritis. Inflamm Res. 2019; 68:597-611.

20 Ouhara K, Munenaga S, Kajiya M, Takeda K, Matsuda S, Sato Y, et al. The induced RNAbinding protein, HuR, targets 3'-UTR region of IL-6 mRNA and enhances its stabilization in periodontitis. Clin Exp Immunol. 2018; 192:325-36.

21 Olsson J, Jacobson TA, Paulsson JM, Dadfar E, Moshfegh A, Jacobson SH, et al. Expression of neutrophil SOD2 is reduced after lipopolysaccharide stimulation: a potential cause of neutrophil dysfunction in chronic kidney disease. Nephrol Dial Transplant. 2011;26:2195201.

22 Ernandez T, Mayadas TN. The changing landscape of renal inflammation. Trends $\mathrm{Mol}$ Med. 2016;22:151-63.

23 Han C, Sun T, Liu Y, Fan G, Zhang W, Liu C. Protective effect of Polygonatum sibiricum polysaccharides on gentamicin-induced acute kidney injury in rats via inhibiting p38 MAPK/ATF2 pathway. Int J Biol Macromol. 2020;151:595-601.

24 Luo F, Shi J, Shi Q, Xu X, Xia Y, He X. Mitogen-activated protein kinases and hypoxic/ ischemic nephropathy. Cell Physiol Biochem. 2016:39:1051-67.

25 Seldin MM, Meng Y, Qi H, Zhu W, Wang Z, Hazen SL, et al. Trimethylamine $\mathrm{N}$-oxide promotes vascular inflammation through signaling of mitogen-activated protein kinase and nuclear factor-кB. J Am Heart Assoc. 2016;5: e002767.
26 Tanaka H, Iwasaki Y, Yamato H, Mori Y, Komaba $\mathrm{H}$, Watanabe $\mathrm{H}$, et al. P-cresyl sulfate induces osteoblast dysfunction through activating JNK and p38 MAPK pathways. Bone. 2013;56:347-54.

27 Gurgis FM, Yeung YT, Tang MX, Heng B, Buckland M, Ammit AJ, et al. The p38-MK2HuR pathway potentiates EGFRvIII-IL-1 $\beta$ driven IL-6 secretion in glioblastoma cells. Oncogene. 2015;34:2934-42.

28 Guo J, Lei M, Cheng F, Liu Y, Zhou M, Zheng $\mathrm{W}$, et al. RNA-binding proteins tristetraprolin and human antigen $\mathrm{R}$ are novel modulators of podocyte injury in diabetic kidney disease. Cell Death Dis. 2020;11:413.

29 Sedeek M, Nasrallah R, Touyz RM, Hébert RL. NADPH oxidases, reactive oxygen species, and the kidney: Friend and foe. J Am Soc Nephrol. 2013;24:1512-8.

30 Shi Q, Lee DY, Féliers D, Abboud HE, Bhat MA, Gorin Y. Interplay between RNA-binding protein $\mathrm{HuR}$ and Nox4 as a novel therapeutic target in diabetic kidney disease. $\mathrm{Mol}$ Metab. 2020;36:100968.

31 Zhang X, Li Y, Yang P, Liu X, Lu L, Chen Y, et al. Trimethylamine- $\mathrm{N}$-oxide promotes vascular calcification through activation of NLRP3 (nucleotide-binding domain, leucinerich-containing family, pyrin domain-containing-3) inflammasome and NF-kappaB (nuclear factor kappaB) signals. Arterioscler Thromb Vasc Biol. 2020;40:751-65.

32 Mulay SR. Multifactorial functions of the inflammasome component NLRP3 in pathogenesis of chronic kidney diseases. Kidney Int. 2019;96:58-66.

33 Moon JS, Nakahira K, Chung KP, DeNicola GM, Koo MJ, Pabón MA, et al. NOX4-dependent fatty acid oxidation promotes NLRP3 inflammasome activation in macrophages. Nat Med. 2016;22:1002-12. 